\title{
Euphorbia milii-Endophytic Bacteria Interactions Affect Hormonal Levels of the Native Host Differently Under Various Airborne Pollutants
}

\author{
Gholamreza Khaksar, ${ }^{1}$ Dian Siswanto, ${ }^{1,2}$ Chairat Treesubsuntorn, ${ }^{3}$ and Paitip Thiravetyan ${ }^{1}$ \\ ${ }^{1}$ School of Bioresources and Technology, King Mongkut's University of Technology Thonburi, Bangkok 10150, Thailand; \\ ${ }^{2}$ Faculty of Mathematics and Natural Sciences, Brawijaya University, Malang 65145, Indonesia; and ${ }^{3}$ Pilot Plant Development \\ and Training Institute, King Mongkut's University of Technology Thonburi
}

Accepted 20 July 2016.

\begin{abstract}
This study was conducted to assess the effect of plant-native endophytic bacteria interactions on indole-3-acetic acid (IAA), ethylene levels, and hormonal balance of Euphorbia milii under different airborne pollutants. IAA levels and airborne formaldehyde removal by $E$. milii enhanced when inoculated with endophytic isolates. However, one isolate, designated as root endophyte 4, with the highest levels of IAA production individually, declined gaseous formaldehyde removal of plant, since it disturbed hormonal balance of $E$. milii, leading to IAA levels higher than physiological concentrations, which stimulated ethylene biosynthesis and stomatal closure under light conditions. However, plant-root endophyte 4 interactions favored airborne benzene removal, since benzene was more phytotoxic and the plant needed more IAA to protect against benzene phytotoxicity. As trimethylamine (TMA) was not toxic, it did not affect plant-endophyte interactions. Therefore, IAA levels of root endophyte 4-inoculated $E$. milii was not significantly different from a noninoculated one. Under mixed-pollutant stress (formaldehyde, benzene, TMA), root endophyte 4-inoculated $E$. milii removed benzene at the lowest rate, since benzene was the most phytotoxic pollutant with the greatest molecular mass. However, TMA (with greater molecular mass) was removed faster than formaldehyde due to higher phytotoxicity of formaldehyde. Plantendophyte interactions were affected differently under various airborne pollutants.
\end{abstract}

Taking advantage of plant inoculation with endophytic bacteria is of the utmost importance to address some drawbacks that still affect the feasibility of phytoremediation for indoor air purification. Better understanding of plant-endophytic bacteria interactions under airborne pollutant stress bears great value for both phytoprotection and phytoremediation enhancement. Among plant-associated microorganisms, endophytic bacteria are the ones that can colonize internal parts of the plant tissue without causing any harm (Afzal et al. 2014). Endophytic bacteria can colonize plant tissue via the root zone as the main point of entrance (Kobayashi and Palumbo 2000) and live within intercellular spaces (Patriquin and Döbereiner 1978) or

Corresponding author: P. Thiravetyan; E-mail: paitip.thi@kmutt.ac.th; Telephone: +66-2-470-7535; Fax: +66-2-452-3455.

*The $\boldsymbol{e}$-Xtra logo stands for "electronic extra" and indicates that six supplementary figures and four supplementary tables are published online.

○ 2016 The American Phytopathological Society the vascular system (Bell et al. 1995). Upon inoculation into the plant, endophytic bacteria can confer protection to its host against pollutant phytotoxicity and enhance plant growth and development under pollutant-imposed stress by means of phytohormone production, such as IAA and 1-aminocyclopropane1-carboxylic acid (ACC) deaminase activity (Rajkumar et al. 2009). Under abiotic stress conditions (pollutant-imposed stress), plants need higher levels of IAA to protect against this stress. Thus, plant-endophytic bacteria interactions would provide higher levels of IAA through IAA production by one or both associated or inoculated endophytic bacteria. Numerous successful cases of plant inoculation with endophytic bacteria for both phytoprotection and phytoremediation enhancement have been well documented (Babu et al. 2013; Barac et al. 2004; Ho et al. 2012; Khaksar et al. 2016a and b). However, Xie et al. (1996) reported that wild-type Pseudomonas putida GR12-2, which could produce low IAA levels $\left(2 \mu \mathrm{g} \mathrm{ml}^{-1}\right)$, enhanced root elongation of canola (Brassica napus L.) seedlings. However, the mutant $P$. putida GR12-2/aux1, which could produce $8.2 \mu \mathrm{g} \mathrm{ml}^{-1}$ of IAA, inhibited seedling root growth. This phenomenon was attributed to hormonal cross talk between IAA and ethylene, since IAA at high concentrations would stimulate ACC synthesis through transcriptionally inducing the ACC synthase gene family (Chen et al. 2005). ACC is the precursor of ethylene biosynthesis (Adams and Yang 1979). Phytohormone ethylene is mainly involved in plant biotic and abiotic stress response and also acts as a modulator of plant growth and development (Abeles et al. 1992). Ethylene biosynthesis is stimulated by biotic and abiotic stress factors as well as the presence and concentration of other phytohormones. While low levels of ethylene would induce the transcription of plant defensive and protective genes, higher levels of ethylene inhibit plant growth (Mayak et al. 2004). Ethylene could act as a feedback inhibitor of IAA, inhibiting its biosynthesis or limiting IAA functioning (Li et al. 2013).

To the best of our knowledge, there are no published studies in the literature regarding the impact of native endophytic bacteria inoculation on IAA, ethylene levels, and the hormonal balance of the host plant under airborne pollutant stress. Investigating whether various airborne pollutants would affect plant-endophyte interactions differently seems lacking. Moreover, studying the effect of mixed-pollutant stress on plant-endophyte interactions and hormonal levels of the host plant has not been previously examined.

Euphorbia milii, an ornamental indoor plant, is a flowering species with densely spiny stems. It has been reported that E. milii harbored a remarkable efficiency for trimethylamine (TMA) (an indoor air pollutant) removal, uptaking 100 ppm 
within 16 h (Chhon 2013). Thereafter, this study was conducted to i) inoculate $E$. milii with native endophytic isolates and examine the impact of plant-endophyte interactions on IAA, ethylene levels, and hormonal cross talk between these two hormones under different airborne pollutants, ii) evaluate the impact of different gaseous pollutants on plant-native endophyte interactions for enhancing airborne pollutant removal, and iii) investigate the impact of plant-endophyte interactions on hormonal levels of the host plant under mixed-pollutant stress. In our study, formaldehyde, benzene, and TMA were chosen as model pollutants. Benzene and formaldehyde are human carcinogens that contribute to an important fraction of indoor air pollution, since they are emitted from numerous sources indoors. Furthermore, these pollutants are phytotoxic. TMA is an aliphatic amine gas, at room temperature, with a pungent odor that is associated with bad breath. TMA has a significantly low odor threshold and is discharged at high concentrations at food processing plants and fishery industries, leading to adverse health impacts (Khaksar et al. 2016a and b; Treesubsuntorn and Thiravetyan 2012; Siswanto et al. 2016).

\section{RESULTS AND DISCUSSION}

Airborne formaldehyde removal by isolated bacteria from $E$. milii.

A total of seven formaldehyde-tolerant endophytic bacteria were isolated from E. milii. Gaseous formaldehyde removal efficiency (within $12 \mathrm{~h}$ ) of isolated endophytic bacteria is shown in Figure 1A. Root endophyte 1, root endophyte 3, and leaf endophyte 1 harbored greater gaseous formaldehyde removal efficiency (within $12 \mathrm{~h}$ ) compared with other isolates $(P<0.05)$. It was important to evaluate whether or not the efficiency of gaseous formaldehyde removal by isolated endophytic bacteria could serve as a dependable criterion for choosing the most efficient isolate for plant inoculation.

\section{Effect of isolated endophytic bacteria inoculation} on native $\boldsymbol{E}$. milii for airborne formaldehyde removal.

Each isolated endophytic bacteria was individually inoculated into native hosts through soil to examine the effect of native endophyte inoculation into $E$. milii for the enhancement of airborne formaldehyde removal. Native E. milii inoculated with root endophyte 1 , root endophyte 5 , root endophyte 2 , leaf endophyte 1, and leaf endophyte 2 harbored greater gaseous formaldehyde removal efficiency (within $12 \mathrm{~h}$ ) when compared with noninoculated and other endophyte-inoculated plants $(P<0.05)$ (Fig. 1B). Greater gaseous formaldehyde removal efficiency of these inoculated plants could likely be due to higher levels of IAA within plants that protected E. milii against formaldehyde phytotoxicity and enhanced plant growth and development under formaldehyde stress by lowering the levels of intracellular reactive oxygen species (ROS) (Table 1). The positive role of auxin (IAA) in enhancing antioxidant enzymes activity and controlling ROS accumulation has been well documented (Guan and Scandalios 2002; Fei et al. 2016). Under formaldehyde stress conditions, plants need higher levels of IAA to protect against stress. Therefore, endophytic bacteria would produce IAA to increase IAA levels within its host plant. A higher population of endophytic bacteria within an endophyte-inoculated plant (compared with a noninoculated one) would contribute to higher IAA levels under stress conditions, which could protect the plant against formaldehyde phytotoxicity.

Quantification by quantitative polymerase chain reaction (qPCR) of each endophytic inoculum within an endophyteinoculated plant indicated a significant increase in $16 \mathrm{~S}$ ribosomal RNA (rRNA) gene copy numbers of that endophytic bacterium compared with a noninoculated one $(P<0.05)$ (Fig. $2)$. This result confirmed the larger population of the endophytic inoculum within the endophyte-inoculated plant. Under normal conditions (without formaldehyde), endophyte inoculation into the plant did not trigger any stress response, such as ethylene production or greater intracellular ROS accumulation (Table 1). However, root endophyte 3 inoculation into the plant stimulated a stress response, such as ethylene production, and greater ROS accumulation without formaldehyde (Table 1). This phenomenon indicated that a greater population of root endophyte 3 within endophyte-inoculated plant would cause stress conditions as a result of unbalancing the endophytic population (root endophyte 3 had a significantly lower population within noninoculated plants compared with other endophytic isolates) (Fig. 2). Thus, root endophyte 3-inoculated E. milii did not have a significantly higher gaseous formaldehyde removal compared with a noninoculated one $(P<0.05)$ (Fig. $1 \mathrm{~B})$, although root endophyte 3 possessed a remarkable efficiency for gaseous formaldehyde removal individually $(93.5 \% \pm$ $0.18 \%$ within $12 \mathrm{~h}$ ). Moreover, native $E$. milii inoculated with root endophyte 4 possessed a significantly lower gaseous
A

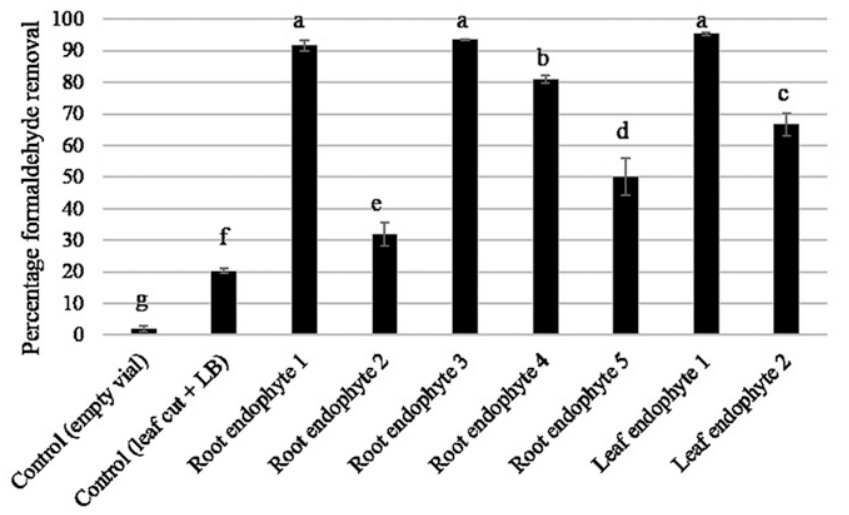

B

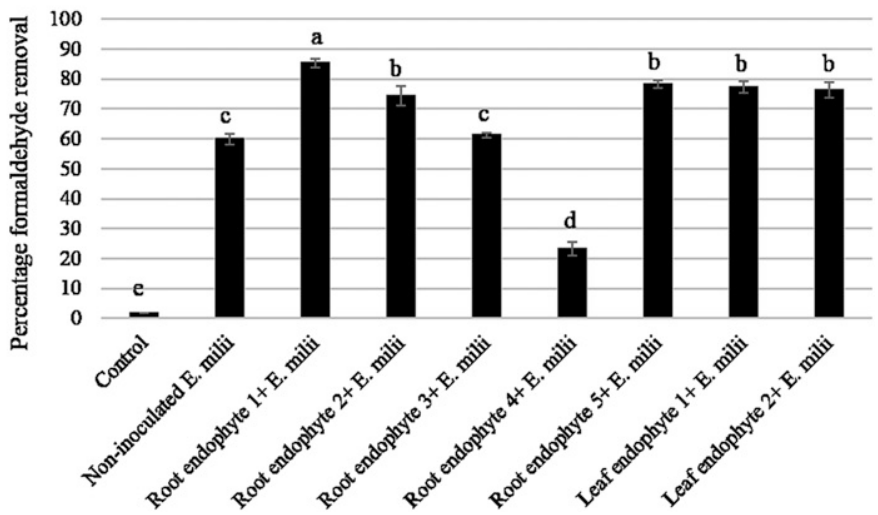

Fig. 1. A, Percentage of gaseous formaldehyde removed by endophytic isolates in volatile organic analysis vials at $12 \mathrm{~h}$ (initial formaldehyde concentration, $100 \mathrm{ppm}$ ). B, Percentage of gaseous formaldehyde removed by endophyte-inoculated and noninoculated Euphorbia milii in glass chambers at $12 \mathrm{~h}$ (initial formaldehyde concentration, $20 \mathrm{ppm})$. Bars represent standard deviation (SD) of three replications. Values with different letters are significantly different $(P<$ $0.05)$. Control is a pot with soil and inoculum covered with aluminum foil. 
formaldehyde removal rate, when compared with a noninoculated plant $(P<0.05)$ (Fig. 1B). However, root endophyte 4 harbored a considerable potential for removing gaseous formaldehyde individually (percentage removal of $80.93 \% \pm 1.20 \%$ within $12 \mathrm{~h}$ ).
Accordingly, individual characteristics of endophytic isolates, such as gaseous formaldehyde removal efficiency, might not serve as a dependable criterion for choosing the most efficient isolate for successful native host inoculation.

Table 1. Removal levels of gaseous formaldehyde, indole-3-acetic acid (IAA), ethylene, and reactive oxygen species (ROS) by endophyte-inoculated and noninoculated Euphorbia milii ${ }^{\mathrm{Z}}$

\begin{tabular}{|c|c|c|c|c|c|c|c|c|}
\hline \multirow[b]{2}{*}{ Type } & \multicolumn{2}{|c|}{ \% Removal } & \multicolumn{2}{|c|}{ IAA levels $\left(\mu g g^{-1} F W\right)$} & \multicolumn{2}{|c|}{$\begin{array}{l}\text { Ethylene levels } \\
\left(\text { nmole } \mathrm{g}^{-1} \text { FW) }\right.\end{array}$} & \multicolumn{2}{|c|}{ ROS levels $\left(\mu M g^{-1}\right.$ FW) } \\
\hline & $12 \mathrm{~h}$ & $48 \mathrm{~h}$ & With FA & Without FA & With FA & Without FA & With FA & Without FA \\
\hline Noninoculated E. milii & $59.93 \pm 1.91 \mathrm{c}$ & $100.00 \pm 0.00 \mathrm{a}$ & $0.04 \pm 0.002 \mathrm{~d}$ & $0.06 \pm 0.003 \mathrm{a}$ & $92.78 \pm 6.40 \mathrm{~b}$ & ND & $524.64 \pm 17.73 b$ & $134.08 \pm 21.07 \mathrm{~b}$ \\
\hline $\begin{array}{l}\text { Root endophyte 1- } \\
\text { inoculated E. milii }\end{array}$ & $85.32 \pm 1.55 \mathrm{a}$ & $100.00 \pm 0.00 \mathrm{a}$ & $0.32 \pm 0.01 b$ & $0.05 \pm 0.001 \mathrm{a}$ & ND & ND & $165.86 \pm 9.86 \mathrm{e}$ & $143.08 \pm 11.99 b$ \\
\hline $\begin{array}{l}\text { Root endophyte 2- } \\
\text { inoculated E. milii }\end{array}$ & $74.43 \pm 1.19 b$ & $100.00 \pm 0.00 \mathrm{a}$ & $0.22 \pm 0.03 c$ & $0.05 \pm 0.002 \mathrm{a}$ & ND & ND & $244.71 \pm 10.03 c$ & $130.97 \pm 21.99 b$ \\
\hline $\begin{array}{l}\text { Root endophyte 3- } \\
\text { inoculated E. milii }\end{array}$ & $61.32 \pm 0.78 c$ & $100.00 \pm 0.00 \mathrm{a}$ & ND & ND & $100.92 \pm 7.03 b$ & $43.77 \pm 4.03 \mathrm{a}$ & $492.03 \pm 11.03 b$ & $256.97 \pm 11.94 \mathrm{a}$ \\
\hline $\begin{array}{l}\text { Root endophyte } 4- \\
\text { inoculated E. milii } \\
\text { Root endophyte 5- }\end{array}$ & $23.21 \pm 2.16 \mathrm{~d}$ & $31.40 \pm 1.07 \mathrm{~b}$ & $0.73 \pm 0.02 \mathrm{a}$ & $0.06 \pm 0.001 \mathrm{a}$ & $278.50 \pm 4.30 \mathrm{a}$ & ND & $834.43 \pm 20.06 a$ & $145.08 \pm 12.06 b$ \\
\hline $\begin{array}{l}\text { inoculated E. milii } \\
\text { Leaf endophyte } 1-\end{array}$ & $78.28 \pm 3.30 \mathrm{~b}$ & $100.00 \pm 0.00 \mathrm{a}$ & $0.34 \pm 0.02 b$ & $0.06 \pm 0.009 \mathrm{a}$ & ND & ND & $211.64 \pm 8.06 \mathrm{~d}$ & $129.07 \pm 12.99 \mathrm{~b}$ \\
\hline $\begin{array}{l}\text { inoculated E. milii } \\
\text { Leaf endophyte 2- }\end{array}$ & $77.32 \pm 1.90 \mathrm{~b}$ & $100.00 \pm 0.00 \mathrm{a}$ & $0.29 \pm 0.03 b$ & $0.05 \pm 0.002 \mathrm{a}$ & ND & ND & $221.96 \pm 10.96 \mathrm{~cd}$ & $136.81 \pm 9.86 b$ \\
\hline inoculated E. milii & $76.32 \pm 2.60 \mathrm{~b}$ & $100.00 \pm 0.00 \mathrm{a}$ & $0.20 \pm 0.04 c$ & $0.07 \pm 0.008 \mathrm{a}$ & ND & ND & $233.08 \pm 11.97 \mathrm{c}$ & $141.71 \pm 11.55 b$ \\
\hline
\end{tabular}

${ }^{\mathrm{z}}$ Percent removal measured within 12 and $48 \mathrm{~h}$ of fumigation and at the end of fumigation period ( $48 \mathrm{~h}$ ) (IAA, ethylene, and ROS), under an initial gaseous formaldehyde concentration of $20 \mathrm{ppm}$. Data are listed as average \pm standard deviation for three replications. Values in the same column with different letters are significantly different $(P<0.05)$. ND $=$ not detected, $\mathrm{FW}=$ fresh weight, $\mathrm{FA}=$ formaldehyde.

A

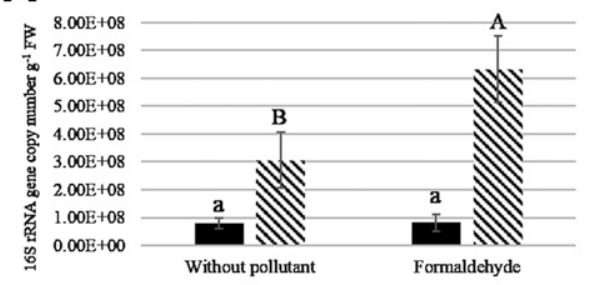

D - Non-inoculated plant NRoot endophyte 1-inoculated plant

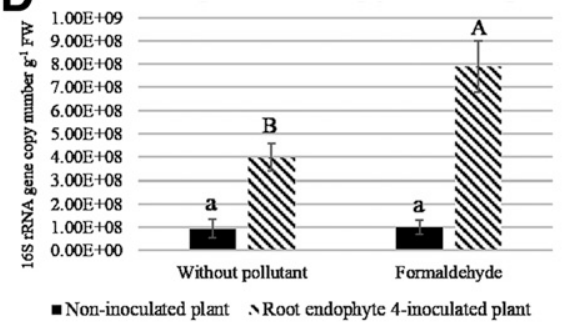

G

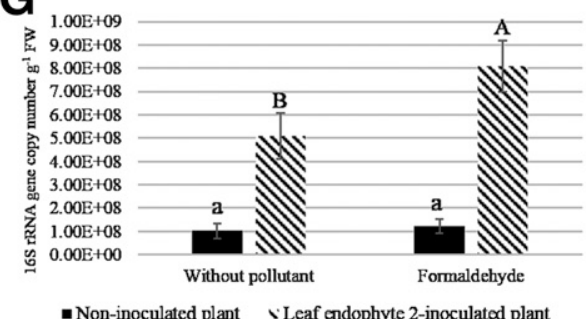

B

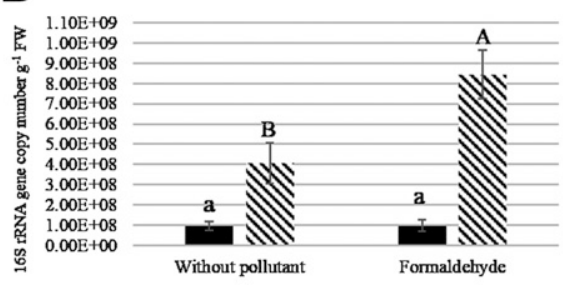

E

- Non-inoculated plant S Root endophyte 2-inoculated plant

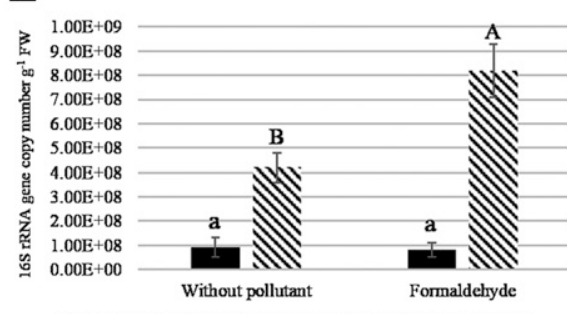

- Non-inoculated plant - Root endophyte 5-inoculated plant

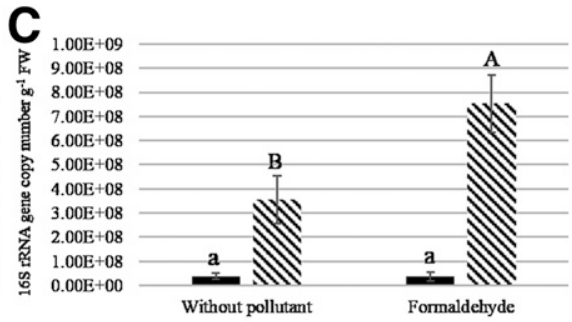

$\mathbf{F}$

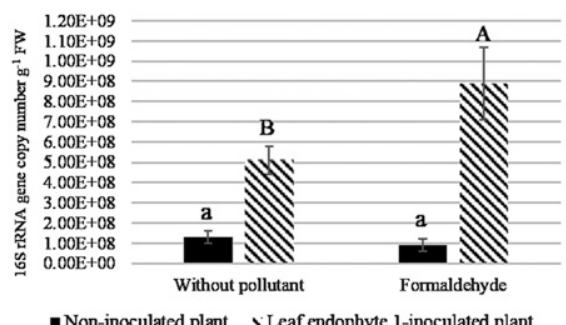

Fig. 2. 16s rRNA gene copy number per gram of fresh weight of A, root endophyte 1-, B, root endophyte 2-, C, root endophyte 3-, D, root endophyte 4-, E, root endophyte 5-, F, leaf endophyte 1-, and $\mathbf{G}$, leaf endophyte 2-inoculated and noninoculated plants in the presence and absence of formaldehyde at the end of the fumigation cycle ( $48 \mathrm{~h}$ ) (4 days after inoculation). Bars indicate standard deviation of three replications. For each inoculum, lower case letters represent comparisons made between noninoculated plants with and without formaldehyde (same letters are not significantly different $[P<0.05]$ ), and upper case letters represent comparisons made between endophyte-inoculated plants with and without formaldehyde (same letters are not significantly different $[P<0.05])$. FW $=$ fresh weight. 
Native $E$. milii-root endophyte 4 interactions disturbed hormonal balance of plants under airborne formaldehyde stress.

Root endophyte 4 inoculation into the plant did not stimulate any stress response in the absence of formaldehyde (Table 1). However, this inoculation reduced gaseous formaldehyde removal by the native host plant. One possible factor might be correlated to hormonal balance of the endophyte-inoculated E. milii, as endophyte inoculation could alter hormonal levels of the host plant. Root endophyte 4 has been proven to produce $64.32 \pm 2.94 \mathrm{mg}$ of IAA per liter, which was significantly more than that of other isolated endophytic bacteria $(P<0.05)$ (Supplementary Table S1). IAA levels were measured at $0.73 \pm 0.02 \mu \mathrm{g}$ per gram of fresh weight of root endophyte 4-inoculated plant, which might be a higher level than physiological concentrations and could stimulate ethylene biosynthesis (Table 1). Accordingly, ethylene levels were found at $278.50 \pm 4.30$ nmole per gram of fresh weight of $E$. milii inoculated with root endophyte 4 . In contrast, ethylene levels could not be detected in E. milii inoculated with root endophyte 1 , with IAA levels at $0.32 \pm 0.01 \mu \mathrm{g}$ per gram of fresh weight of root endophyte 1-inoculated plant (Table 1). This phenomenon manifested the advantageous role of phytohormone IAA at physiological levels, which protected its native host against formaldehyde phytotoxicity (Supplementary Fig. S1A) and enhanced gaseous formaldehyde removal of its native host (Table 1). In contrast, IAA levels greater than physiological concentrations could stimulate ethylene biosynthesis at high levels, leading to growth inhibition and stress-mediated impacts and, thus, inferior gaseous formaldehyde removal (Table 1). No signs of formaldehyde phytotoxicity were observed in noninoculated E. milii under formaldehyde stress. Moreover, Daszkowska-Golec and Szarejko (2013) reported that IAA levels at physiological concentrations could induce stomatal opening, whereas levels higher than physiological concentrations would act the opposite. Accordingly, patterns of stomata were observed at $48 \mathrm{~h}$ of fumigation during the day time (light conditions). Stomata were open for noninoculated (Fig. 3A) and all endophyte-inoculated plants (Fig. 3B to G), except for the root endophyte 4-inoculated plant, which had closed stomata (Fig. $3 \mathrm{H}$ ). Inoculating root endophyte 4 into a native host might contribute to IAA levels higher than physiological concentrations, which stimulated ethylene biosynthesis and stomatal closure under light conditions. Hence, gaseous formaldehyde removal of root endophyte 4-inoculated E. milii declined compared with noninoculated and other endophyteinoculated plants. In the absence of formaldehyde, stomata were observed to be open for noninoculated and endophyte- inoculated plants (Supplementary Fig. S2). Root endophyte 1 and root endophyte 4 have been identified as Acinetobacter venetianus MTCC11369 and Citrobacter amalonaticus Y19 by $16 \mathrm{~S}$ rRNA gene sequencing, respectively (Supplementary Table S2).

\section{Native $E$. milii-endophytic bacteria interactions under different airborne pollutants: the role of IAA and ethylene.}

Root endophyte 1, which enhanced gaseous formaldehyde removal of the native host, and root endophyte 4, which impaired gaseous formaldehyde removal, were chosen for plant inoculation to further investigate whether they would behave likewise under different airborne pollutants (benzene and TMA) or not. Interestingly, the results revealed that E. milii inoculated with root endophyte 4 harbored the highest airborne benzene removal efficiency, as compared with inoculated plants with root endophyte 1 and noninoculated ones $(P<0.05)$ (Fig. $4 \mathrm{~A})$. Furthermore, root endophyte 4-inoculated plant harbored a significantly higher TMA removal efficiency compared with plants inoculated with root endophyte 1 and noninoculated plants (Fig. 4B), which was in contrast with formaldehyde stress (plants inoculated with root endophyte 4 possessed a significantly lower gaseous formaldehyde removal compared with plants inoculated with root endophyte 1) (Fig. 4C).

Different removal efficiencies of plants inoculated with root endophytes 1 and 4 under various airborne pollutants could be linked to the impact of various airborne pollutants with different phytotoxicity levels on plant-endophyte interactions and hormonal balance of the inoculated plant. Upon endophyte inoculation into the plant, plant IAA levels would increase (within physiological levels), which could confer protection to the host against pollutant phytotoxicity and stimulate stomatal opening and, hence, better airborne pollutant removal. However, when levels of IAA reach concentrations higher than physiological levels, it could inhibit plant growth and stimulate ethylene biosynthesis and stomatal closure, which could lead to stress-mediated impacts and lower airborne pollutant removal capability. Successful plant inoculation for phytoremediation enhancement depends on not only individual characteristics of endophytic bacteria, such as levels of IAA production and gaseous pollutant removal efficiency, but also plant-endophyte interactions and its impact on hormonal balance of the plant, which is affected differently by various airborne pollutants of different phytotoxicity levels.

Benzene, formaldehyde, and TMA have different levels of phytotoxicity. Accordingly, ethylene levels were measured at $150.72 \pm 3.86$ nmole per gram of fresh weight of noninoculated
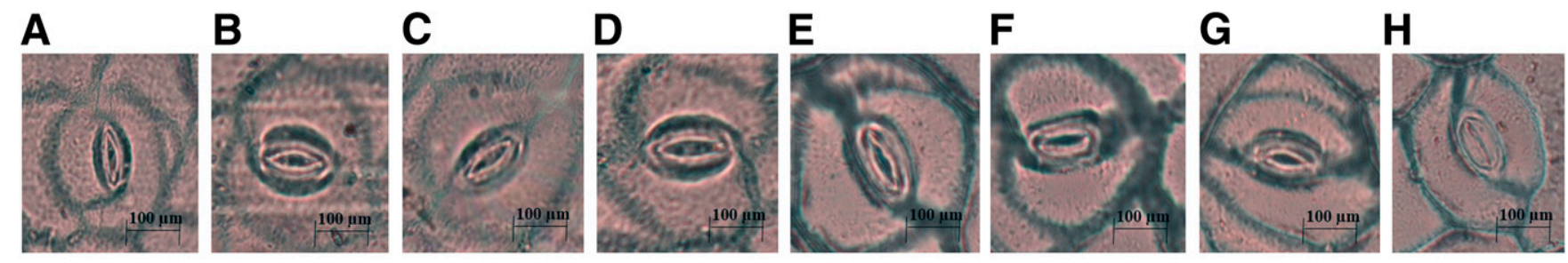
A= Non-inoculated $E$. milii
$\mathrm{E}=$ Root endophyte 5-inoculated $E$. milii
B= Root endophyte 1-inoculated $E$. milii
F= Leaf endophyte 1-inoculated $E$. milii
C= Root endophyte 2-inoculated $E$. milii
G= Leaf endophyte 2-inoculated $E$. milii
D= Root endophyte 3-inoculated $E$. milii
H= Root endophyte 4-inoculated $E$. milii

Fig. 3. Stomatal patterns of A, noninoculated and $\mathbf{B}$ to $\mathbf{H}$, endophyte-inoculated Euphorbia milii under formaldehyde stress, observed under light conditions (day time). Pictures of representative stomata were obtained at $48 \mathrm{~h}$ of fumigation. 
E. milii under benzene stress, which was significantly higher than ethylene levels of noninoculated E. milii under formaldehyde stress $(92.78 \pm 6.40$ nmole per gram of fresh weight $)$ and TMA (not detected) $(P<0.05)$ (Table 2). Moreover, it took a much longer time for noninoculated $E$. milii to remove 20 ppm of benzene $(96 \mathrm{~h})$ compared with formaldehyde $(20 \mathrm{ppm}$ within $48 \mathrm{~h}$ ) and TMA (100 ppm within $16 \mathrm{~h}$ ) (Fig. 4), which might indicate that benzene is the most phytotoxic compound. Benzene and formaldehyde were toxic to E. milii, thus posing stress to the plant. Therefore, plants would need higher levels of IAA to protect against pollutant stress. Under these stress conditions, root endophyte 4-inoculated plants gained the benefit of endophyte inoculation and pushed endophytic bacteria to produce IAA at high levels, which favored airborne benzene removal efficiency, since plants were under high stress conditions and needed high amounts of IAA to protect against this stress. As root endophyte 4 could produce IAA at significantly higher levels (compared with root endophyte 1), root endophyte 4-plant interactions could provide higher levels of IAA to protect against benzene phytotoxicity (compared with root endophyte 1-plant interactions). Consequently, root endophyte 4-inoculated plants had a higher airborne benzene removal efficiency compared with root endophyte 1-inoculated plants $(P<0.05)$ (Table 2$)$. However, IAA levels were measured at $0.73 \pm 0.02 \mu \mathrm{g}$ per gram of fresh weight of root endophyte 4-inoculated plants under formaldehyde stress,

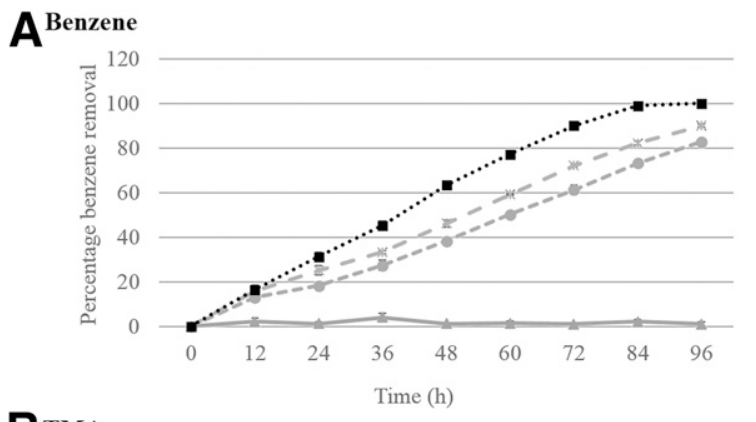

B TMA

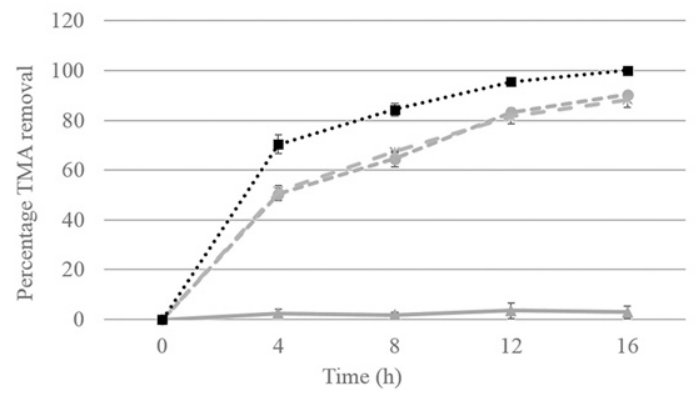

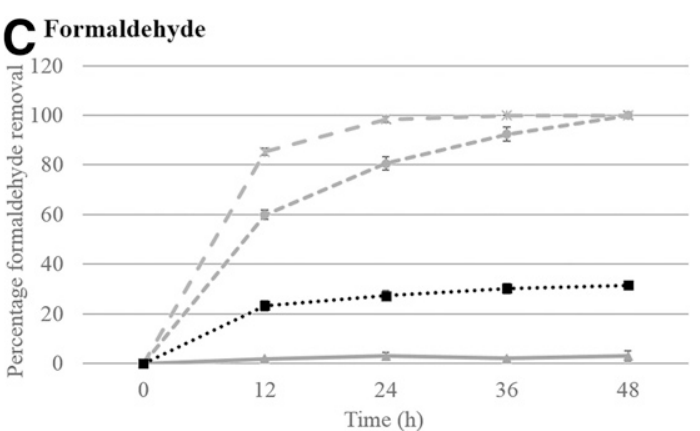

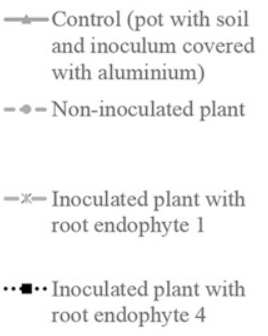

Fig. 4. Percentage of airborne A, benzene, B, trimethylamine (TMA), and C, formaldehyde removed by noninoculated and endophyte-inoculated Euphorbia milii in a glass chamber. The data are presented as the mean \pm standard deviation of three replications.

Table 2. Percentage of pollutant, indole-3-acetic acid (IAA), ethylene, and reactive oxygen species (ROS) levels removed by endophyte-inoculated and noninoculated Euphorbia milii ${ }^{\mathrm{z}}$

\begin{tabular}{|c|c|c|c|}
\hline Type & Noninoculated $E$. milii & Root endophyte 1-inoculated $E$. milii & Root endophyte 4-inoculated $E$. milii \\
\hline \multicolumn{4}{|l|}{ Benzene (96 h) } \\
\hline$\%$ removal & $70.23 \pm 2.34 c$ & $84.34 \pm 1.09 b$ & $100.00 \pm 0.00 \mathrm{a}$ \\
\hline IAA levels & ND & $0.12 \pm 0.02 b$ & $0.36 \pm 0.01 \mathrm{a}$ \\
\hline Ethylene levels & $150.72 \pm 3.86 \mathrm{a}$ & $30.49 \pm 2.08 b$ & ND \\
\hline ROS levels & $602.87 \pm 14.31 \mathrm{a}$ & $365.72 \pm 18.94 b$ & $211.06 \pm 10.05 \mathrm{c}$ \\
\hline \multicolumn{4}{|c|}{ Formaldehyde (48 h) } \\
\hline$\%$ removal & $100.00 \pm 0.00 \mathrm{a}$ & $100.00 \pm 0.00 \mathrm{a}$ & $31.40 \pm 1.07 \mathrm{~b}$ \\
\hline IAA levels & $0.04 \pm 0.002 \mathrm{c}$ & $0.32 \pm 0.01 b$ & $0.73 \pm 0.02 \mathrm{a}$ \\
\hline Ethylene levels & $92.78 \pm 6.40 \mathrm{~b}$ & ND & $278.50 \pm 4.30 \mathrm{a}$ \\
\hline ROS levels & $524.64 \pm 17.73 b$ & $165.86 \pm 9.86 c$ & $834.43 \pm 20.06 a$ \\
\hline \multicolumn{4}{|l|}{ TMA (16 h) } \\
\hline$\%$ removal & $90.32 \pm 1.08 \mathrm{~b}$ & $88.32 \pm 3.21 b$ & $100.00 \pm 0.00 \mathrm{a}$ \\
\hline IAA levels & $0.05 \pm 0.009 \mathrm{a}$ & $0.06 \pm 0.001 \mathrm{a}$ & $0.06 \pm 0.002 \mathrm{a}$ \\
\hline Ethylene levels & ND & ND & ND \\
\hline ROS levels & $194.84 \pm 8.05 a$ & $183.05 \pm 11.06 \mathrm{a}$ & $201.05 \pm 20.64 \mathrm{a}$ \\
\hline \multicolumn{4}{|l|}{ Without pollutant } \\
\hline$\%$ removal & - & - & - \\
\hline IAA levels & $0.06 \pm 0.004 \mathrm{a}$ & $0.08 \pm 0.009 a$ & $0.07 \pm 0.011 \mathrm{a}$ \\
\hline Ethylene levels & ND & ND & ND \\
\hline ROS levels & $134.08 \pm 21.07 \mathrm{a}$ & $156.95 \pm 11.07 \mathrm{a}$ & $142.51 \pm 21.94 \mathrm{a}$ \\
\hline
\end{tabular}

\footnotetext{
${ }^{\mathrm{z}}$ Measured at the end of fumigation period under benzene $(96 \mathrm{~h})$, formaldehyde $(48 \mathrm{~h})$, and trimethylamine (TMA) $(16 \mathrm{~h})$. Data are listed as average \pm standard deviation for three replications. Values in the same row with different letters are significantly different $(P<0.05)$. Values per gram of fresh weight are as follows: IAA, $\mu \mathrm{g} \mathrm{g}^{-1}$; ethylene, nmole $\mathrm{g}^{-1}$; ROS, $\mu \mathrm{M} \mathrm{g}^{-1}$. ND = not detected.
} 
which was higher than physiological needs of the plant, owing to the fact that formaldehyde was less toxic (compared with benzene) and plants did not need high amounts of IAA. These levels of IAA, higher than the plant's physiological needs, stimulated ethylene biosynthesis at high levels and stomatal closure under light conditions. Therefore, root endophyte 4 inoculated plants harbored a lower airborne formaldehyde removal efficiency compared with root endophyte 1-inoculated plants $(P<0.05)$ (Table 2$)$.

As TMA was not toxic to plants, it did not affect plantendophyte interactions. Thus, hormonal balance of the inoculated plant was not significantly changed. IAA levels were measured at $0.06 \pm 0.002 \mu \mathrm{g}$ per gram of fresh weight of inoculated plant with root endophyte 4 , which was not significantly higher than noninoculated and root endophyte 1-inoculated plants (Table 2). Higher TMA removal efficiency of root endophyte 4-inoculated plants could likely be due to higher populations of root endophyte 4 within inoculated plants (Fig. 5), which increased the TMA diffusion rate through plant tissue (higher than root endophyte 1), owing to the higher TMA removal efficiency of root endophyte 4 individually compared with root endophyte 1 (root endophyte 4 harbored a significantly higher TMA removal efficiency individually $[95.96 \% \pm$ $3.06 \%$ of $100 \mathrm{ppm}$ within $12 \mathrm{~h}$ ] compared with root endophyte 1 $[70.66 \% \pm 2.87 \%$ of $100 \mathrm{ppm}$ within $12 \mathrm{~h}]$ ).

qPCR quantification of root endophyte 1 and root endophyte 4 within endophyte-inoculated plants showed a significant increase in copy numbers of $16 \mathrm{~S}$ rRNA gene, indicating the higher population of root endophyte 1 and root endophyte 4 within inoculated plants compared with a noninoculated one (Fig. 5). Notably, the population of root endophyte 1 within root endophyte 1-inoculated plants was not significantly different from the population of root endophyte 4 within root endophyte 4-inoculated plants in the presence and absence of pollutant (Fig. 5). This observation revealed that the population of the endophytic inoculum within inoculated plant does not play any role in different removal efficiencies and hormonal levels of root endophyte 1 - and root endophyte 4 -inoculated plants under various airborne pollutants. It is noteworthy to mention that the population of the endophytic inoculums within endophyteinoculated plants were significantly lower under no-pollutant and TMA conditions compared with formaldehyde and benzene conditions (Fig. 5). This observation could indicate that, under normal conditions (without stress), endophyte-inoculated plant would balance the population of its endophytic community, since it does not need a higher population of endophytic inoculum.

A proposed model for the role of IAA has been illustrated in Supplementary Fig. S3 (IAA at physiological levels). Under formaldehyde stress (abiotic stress), intracellular ROS production is induced through NADPH oxidase activation (Torres et al. 2002). ROS accumulation would start activating two key enzymes of ethylene biosynthesis, ACC synthase and ACC oxidase.

ACC oxidase would convert the existing pool of ACC to ethylene, which in turn, stimulates the plant protective/defensive response (this ethylene has been referred to as the first peak of ethylene). As ROS accumulation increases, ACC production by ACC synthase and its oxidation to ethylene by ACC oxidase would increase, leading to high levels of ethylene production (the second peak of ethylene), which would be inhibitory to plant growth and magnify stress-mediated impacts (Glick 2014). Upon plant inoculation with endophyte, this inhibitory ethylene production could be controlled. Increased IAA levels within physiological concentrations would protect the plant against pollutant-imposed stress by lowering the levels of intracellular ROS. This phenomenon (controlling and lowering ROS accumulation) would limit the activation of ACC synthase and ACC oxidase enzymes by ROS (Supplementary Fig. S4) (ROS would activate ACC synthase and ACC oxidase in a dose-dependent manner) (Song et al. 2007).

As the activation of ACC synthase enzyme has been limited, ACC synthesis from $S$-adenosylmethionine would be controlled. Moreover, accumulated ACC would be irreversibly hydrolyzed by bacterial ACC deaminase to ammonia and alpha keto butyrate, since ACC oxidase activation has been limited (ACC accumulation was due to the fact that under stress conditions, IAA levels would increase, leading to upregulating the transcription of auxin-responsive genes such as the ACC synthase gene family). Therefore, the levels of inhibitory ethylene production would be controlled and endophyte-inoculated plants would be protected against stress-mediated impacts of formaldehyde.

In contrast, IAA levels greater than physiological concentrations would not protect the plant against formaldehyde stress, since high levels of IAA would enhance ROS production at high levels (Table 2) (auxin [IAA] at physiological levels would induce the production of intracellular ROS at low levels to act as signaling molecules and mediate auxin physiological effects; auxin induces ROS production in a dose-dependent manner) (Song et al. 2007). High levels of
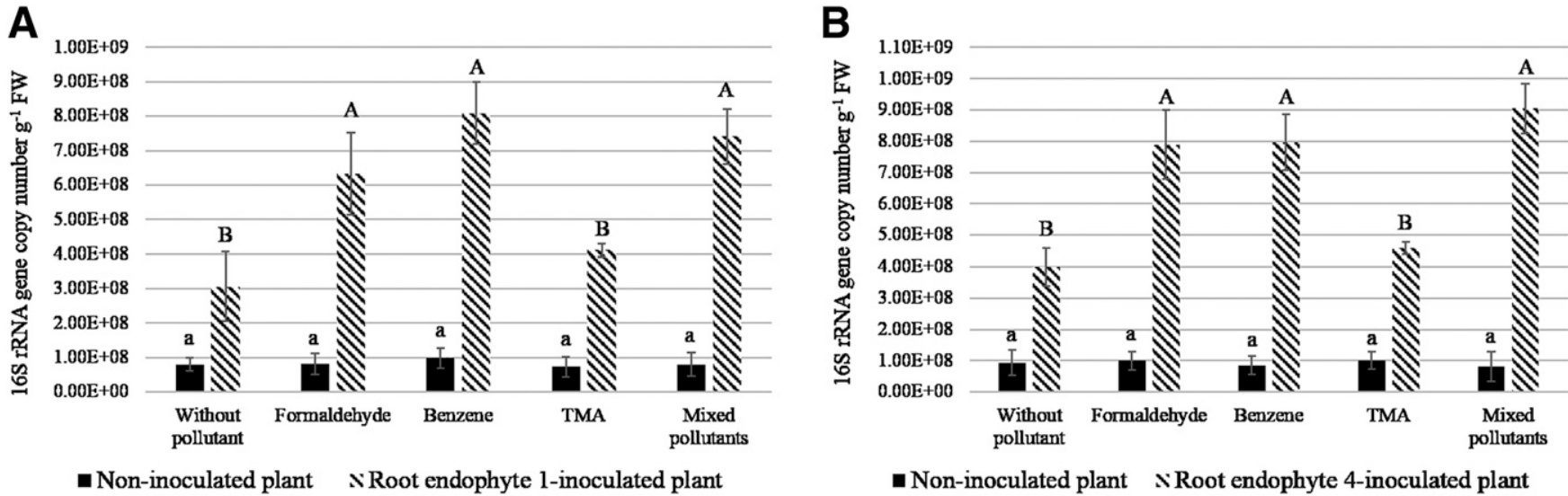

Fig. 5. 16s rRNA gene copy number per gram of fresh weight of A, root endophyte 1- and B, root endophyte 4-inoculated and noninoculated plants in the presence and absence of different pollutants at $48 \mathrm{~h}$ of fumigation cycle (4 days after inoculation). Bars indicate standard deviation of three replications. For each inoculum, comparisons have been made between noninoculated plants with and without pollutants (the same lower case letters are not significantly different $[P<0.05])$. For each inoculum, comparisons have also been made between endophyte-inoculated plants with and without pollutants (the same upper case letters are not significantly different $[P<0.05]) . \mathrm{FW}=$ fresh weight. 
ROS would intensively upregulate the activity of ACC synthase and ACC oxidase enzymes. Moreover, high levels of IAA would transcriptionally induce the ACC synthase gene family at high levels. Hence, large concentrations of ACC would be accumulated.

Most of the accumulated ACC would be oxidized to ethylene by ACC oxidase, due to the fact that ACC oxidase has more affinity to ACC compared with ACC deaminase (Glick 2014). In other words, most of the ACC would be converted to ethylene by ACC oxidase because ACC oxidase has been activated and has more affinity to ACC, leading to high levels of ethylene biosynthesis, which would be inhibitory to plant growth and would intensify formaldehyde stress impacts (Supplementary Fig. S5).

Native $\boldsymbol{E}$. milii-endophytic bacteria interactions under mixed-pollutant stress (benzene + formaldehyde + TMA).

Interestingly, under mixed-pollutant stress, noninoculated E. milii harbored a significantly lower pollutant removal efficiency for each airborne pollutant (Fig. 6A) compared with noninoculated $E$. milii under single-pollutant (Fig. 4) and endophyte-inoculated plants $(P<0.05)$ (Fig. 6B and C). On the contrary, root endophyte 4-inoculated plants possessed a significantly higher removal efficiency rate for each gaseous pollutant compared with root endophyte 1-inoculated and noninoculated plants $(P<0.05)$. IAA was not detected for noninoculated E. milii under mixed-pollutant stress (Table 3). In addition, ethylene levels reached $329.01 \pm 3.98$ nmole per gram of fresh weight of noninoculated plant (Table 3), which was significantly higher than ethylene levels of noninoculated plants under benzene stress $(150.72 \pm 3.86$ nmole per gram of fresh weight) $(P<0.05)$ (Table 2$)$, indicating higher phytotoxicity of mixed pollutants. The highest levels of intracellular

\section{A Non-inoculated plant}

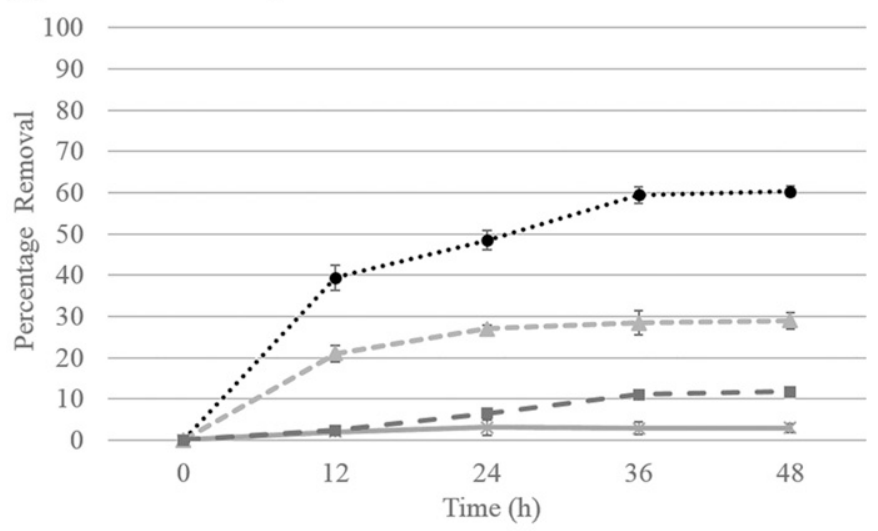

C Root endophyte 4-inoculated plant

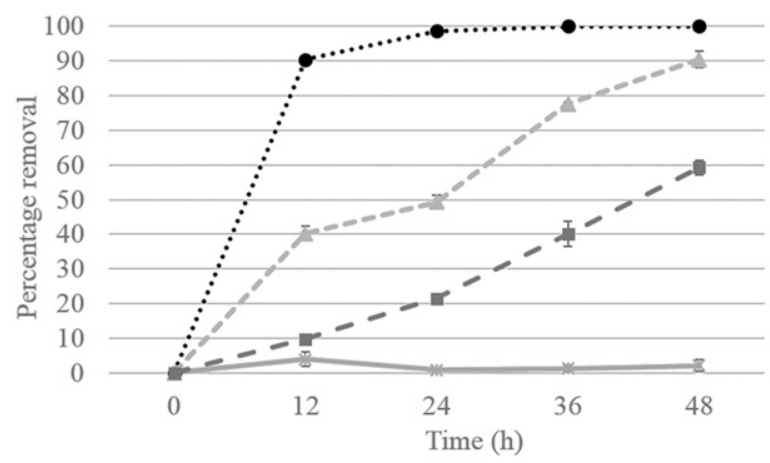

ROS were accumulated within noninoculated plants, whereas the lowest levels of ROS were measured from root endophyte 4 -inoculated plants under mixed-pollutant stress $(P<0.05)$ (Table 3). Phytotoxic-mediated impacts of mixed pollutants were clearly observed for noninoculated $E$. milii at $48 \mathrm{~h}$ of fumigation (Supplementary Fig. S6A). However, no signs of pollutant phytotoxicity were observed for endophyte-inoculated plants, due to higher levels of IAA within endophyte-inoculated plants compared with noninoculated ones. Moreover, patterns of stomata were observed closed for noninoculated plants under light conditions (day time), whereas stomatal patterns of endophyte-inoculated plants were open under light conditions (day time) at $48 \mathrm{~h}$ of fumigation. Under stress conditions of mixed pollutants, root endophyte 4-plant interactions provided higher levels of IAA, which protected the plant against pollutant phytotoxicity and stimulated stomatal opening under light conditions (day time) and, thus, higher pollutant removal efficiency. Among the pollutants, TMA was removed much faster compared with benzene and formaldehyde $(P<0.05)$ (Fig. 6). However, initial concentration of TMA was much higher (100 ppm) compared with benzene and formaldehyde (20 ppm).

One main factor that affects pollutant removal rate is pollutant molecular mass. Sriprapat and Thiravetyan (2013) indicated that a gaseous pollutant with a lower molecular mass would be removed faster than the one with a higher molecular mass. The molecular mass of benzene is $78.11 \mathrm{~g} \mathrm{~mol}^{-1}$, which is higher than the molecular mass of TMA $\left(59.11 \mathrm{~g} \mathrm{~mol}^{-1}\right)$ and formaldehyde $\left(30.03 \mathrm{~g} \mathrm{~mol}^{-1}\right)$. Based on molecular mass, E. milii could remove benzene at the lowest rate compared with formaldehyde and TMA. However, TMA, with higher molecular mass and initial concentration, was removed much faster compared with formaldehyde (Fig. 6). Thus, another important

\section{B Root endophyte 1-inoculated plant}
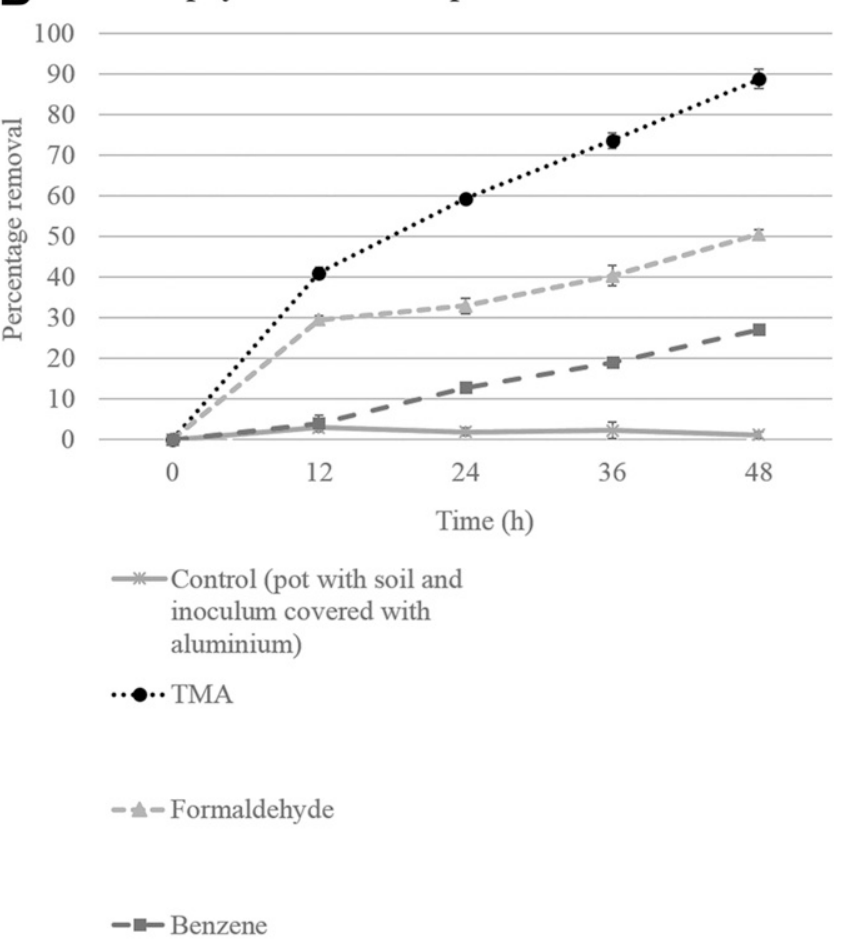

Fig. 6. Percentage of airborne benzene, trimethylamine (TMA), and formaldehyde removed under mixed-pollutant stress by A, noninoculated Euphorbia milii, B, root endophyte 1-inoculated E. milii, and $\mathbf{C}$, root endophyte 4-inoculated E. milii in a glass chamber. The data are presented as the mean \pm standard deviation of three replications. 
factor would influence pollutant removal rate, i.e., the pollutant phytotoxicity level. Higher formaldehyde phytotoxicity led to its lower removal rate compared with TMA, which seemed not to be toxic to E. milii. Moreover, E. milii species is highly efficient for removal of polar pollutants such as TMA (Chhon 2013).

Plant inoculation with native endophytic bacteria could favor airborne pollutant phytoremediation. However, observations should be made to ensure hormonal levels of inoculated plants would not become imbalanced. Plant inoculation with phyllospheric bacteria and its effect on hormonal balance of the host plant under airborne pollutant stress could be the subject of further investigations.

\section{Concluding remarks.}

Native endophytic isolates were individually inoculated into E. milii to investigate the effect of plant-endophyte interactions on hormonal levels and airborne pollutant removal efficiency of native hosts. Notably, root endophyte 4 (Citrobacter amalonaticus Y19) inoculation disturbed the hormonal balance of native hosts, leading to IAA concentrations higher than physiological levels. This phenomenon stimulated ethylene biosynthesis at high levels and stomatal closure under light conditions, resulting in a lower airborne formaldehyde removal level compared with a noninoculated plant. Interestingly, E. milii inoculation with the same isolate favored phytoremediation under airborne benzene, TMA, and mixed-pollutant stress. This phenomenon indicated that plant-endophyte interactions affected hormonal levels of the host plant differently under various airborne pollutants with different phytotoxicity levels. Plant inoculation with endophytic bacteria could be a promising approach to address some drawbacks that continue to affect the feasibility of phytotechnology for indoor air treatment.

\section{MATERIALS AND METHODS}

\section{Plant preparation.}

Four-month-old E. milii plants of similar size were purchased from plant shops in Bangkok, Thailand and were prepared as described in our previous study (Khaksar et al. 2016a).

\section{Isolation and characterization of endophytic bacteria from $E$. milii.}

Before bacteria isolation, plants were kept under $20 \mathrm{ppm}$ of gaseous formaldehyde for eight consecutive days to isolate bacteria that are tolerant to formaldehyde. To isolate endophytic bacteria, samples of leaf and root were washed with distilled water to remove soil and dust particles and were soaked in sterile phosphate-buffered saline $(\mathrm{pH} 7.0)$ for $15 \mathrm{~min}$ to equilibrate osmotic pressure. Thereafter, the root and leaf samples were surface-sterilized according to the method described by Khaksar et al. (2016a). Each surface-sterilized plant sample was macerated in the presence of $3 \mathrm{ml}$ of sterile $10 \mathrm{mM} \mathrm{MgSO} 4$ solution (for $30 \mathrm{~min}$ ) and was ground by a presterilized mortar and pestle. Serial dilutions were spread on Luria-Bertani agar plates and were incubated for 3 days at $32^{\circ} \mathrm{C}$. All morphologically different bacterial colonies were chosen and were subcultured three times to guarantee purity and stability (Visioli et al. 2014). Afterward, isolated endophytic bacteria were screened for the removal efficiency for $100 \mathrm{ppm}$ of gaseous formaldehyde in volatile organic analysis (VOA) vials following the method described by Khaksar et al. (2016a).

\section{IAA production and ACC deaminase activity of endophytic isolates.}

IAA production by endophytic isolates was quantitatively analyzed according to the method described by Gordon and Weber (1951). ACC (1-aminocyclopropane-1-carboxylic acid) deaminase activity of the isolates was examined by measuring the amount of $\alpha$-ketobutyrate ( $\alpha \mathrm{KB}$ ) generated by the enzymatic hydrolysis of ACC. Briefly, each endophytic isolate was grown in Dworkin and Foster (DF) minimal medium supplemented with ACC as the sole nitrogen source for $48 \mathrm{~h}$. Bacterial cells were centrifuged (with a Beckman Coulter Allegra 21R) at $5,000 \mathrm{rpm}$ for $5 \mathrm{~min}$. Cell pellets were then resuspended in $600 \mu \mathrm{l}$ of sterile $10 \mathrm{mM} \mathrm{MgSO}_{4}$ solution. Cells were disrupted by the addition of $30 \mu \mathrm{l}$ of toluene and vigorous vortexing. Afterward, $1 \mathrm{ml}$ of $0.56 \mathrm{~N} \mathrm{HCl}$ was added and the mixture was centrifuged at 5,000 rpm for $5 \mathrm{~min}$, and then, $150 \mu \mathrm{l}$ of $0.1 \%$ 2,4-dinitrophenylhydrazine in $2 \mathrm{~N} \mathrm{HCl}$ was added to $1 \mathrm{ml}$ of the supernatant. The mixture was reacted for $30 \mathrm{~min}$ at $30^{\circ} \mathrm{C}$, was supplemented with $1 \mathrm{ml}$ of $2 \mathrm{~N} \mathrm{NaOH}$, and was assayed for $\alpha$ $\mathrm{KB}$ via determination of the optical density at $540 \mathrm{~nm}$. The concentration was determined based on a standard curve drawn with a series dilution of $\alpha \mathrm{KB}$ (Sigma-Aldrich).

\section{Inoculation of isolated endophytic bacteria into the native host $E$. milii.}

In order to inoculate each endophytic isolate into plants individually, fresh cultures of each endophytic isolate were grown at $32^{\circ} \mathrm{C}$ and $150 \mathrm{rpm}$ on a rotary shaker to an approximate cell concentration of $10^{9}$ cells per milliliter. The bacterial cells were collected by centrifugation at 5,000 rpm for $5 \mathrm{~min}$, were rinsed three times in sterile $10 \mathrm{mM} \mathrm{MgSO}_{4}$ solution, and were suspended in half-strength, sterile Hoagland's nutrient solution to obtain an inoculum density of $10^{9}$ cells per milliliter. Afterward, the inoculum was added to each pot by spraying on the soil surface $(20 \mathrm{ml}$ per pot) (Chen et al. 2013). Three replicates were used for each isolate. As the noninoculated group, three plants were watered with half-strength, sterile Hoagland's nutrient solution ( $20 \mathrm{ml}$ per pot). Then, all of the endophyte-inoculated and noninoculated plants were grown in pots for 2 days under natural conditions (12 h light and $12 \mathrm{~h}$ dark conditions and a constant temperature of $30^{\circ} \mathrm{C}$ ) before doing the fumigation experiment.

Table 3. Indole-3-acetic acid (IAA), ethylene, and reactive oxygen species (ROS) levels of endophyte-inoculated and noninoculated Euphorbia milii ${ }^{2}$

\begin{tabular}{lccc}
\hline Type & Noninoculated $\boldsymbol{E}$. milii & Root endophyte 1-inoculated $\boldsymbol{E}$. milii & Root endophyte 4-inoculated $\boldsymbol{E}$. milii \\
\hline Mixed-pollutant stress & ND & $0.11 \pm 0.01 \mathrm{~b}$ & $0.31 \pm 0.06 \mathrm{a}$ \\
$\quad$ IAA levels & $329.01 \pm 6.98 \mathrm{a}$ & $84.72 \pm 7.98 \mathrm{~b}$ & ND \\
Ethylene levels & $798.11 \pm 24.01 \mathrm{a}$ & $311.06 \pm 15.90 \mathrm{~b}$ & $189.05 \pm 15.15 \mathrm{c}$ \\
ROS levels & $0.07 \pm 0.003 \mathrm{a}$ & $0.07 \pm 0.008 \mathrm{a}$ & $0.08 \pm 0.004 \mathrm{a}$ \\
Without pollutant & ND & ND & ND \\
IAA levels & $128.09 \pm 13.07 \mathrm{a}$ & $142.95 \pm 9.87 \mathrm{a}$ & $139.08 \pm 18.22 \mathrm{a}$ \\
Ethylene levels & ROS levels & & \\
\hline
\end{tabular}

\footnotetext{
${ }^{\mathrm{z}}$ Measured at the end of fumigation period $(48 \mathrm{~h})$ under mixed-pollutant stress. Data are listed as average \pm standard deviation for three replications. Values in

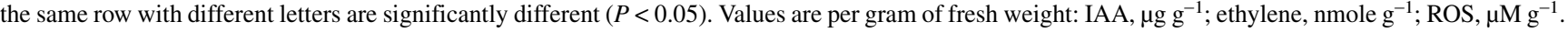
$\mathrm{ND}=$ not detected.
} 


\section{Fumigation experiments.}

Glass chambers, each with a volume of 15.6 liters, were used for fumigation experiments. Each endophyte-inoculated and noninoculated plant was placed in a glass chamber (one plant in one chamber). Three replicates were used for each condition. Before placing the plant inside the chamber, each pot was completely covered with three layers of aluminum foil to avoid the impact of other factors, such as soil and pot adsorption during the fumigation experiment. Each chamber was closed and thoroughly sealed with paraffin tape after placing the plant inside. As the control, a pot containing only soil (no plant control) was sprayed with $20 \mathrm{ml}$ of inoculum, was fully wrapped in three layers of aluminum foil, and was placed in a chamber. In order to ensure gaseous formaldehyde would not be absorbed by the aluminum foil-covered pot, this control was set (three replicates were used for each isolate). Chambers were kept under constant temperature $\left(30^{\circ} \mathrm{C}\right)$ and a pressure of $760 \mathrm{~mm} \mathrm{Hg}$, with $12 \mathrm{~h}$ of alternating light and dark cycles (Sriprapat et al. 2014). A 1.2- $\mu$ l aliquot of liquid formaldehyde (formaldehyde solution, $37 \%$; Sigma-Aldrich, St. Louis) was injected into each chamber to generate an initial concentration of $20 \mathrm{ppm}$ gaseous formaldehyde inside each chamber. Afterward, 0.3-ml samples were obtained from each chamber at $12 \mathrm{~h}$ to analyze the remaining formaldehyde concentration by gas chromatography, with subsequent measurements every $12 \mathrm{~h}$. In our experiment, $20 \mathrm{ppm}$ of gaseous formaldehyde was used as the initial concentration to mimic the real indoor concentrations of formaldehyde found at a real site (Khaksar et al. 2016a).

\section{IAA, ethylene levels, and stomata patterns}

from endophyte-inoculated and noninoculated $E$. milii.

Levels of phytohormone IAA from endophyte-inoculated and noninoculated E. milii was assessed at the end of the fumigation period ( $48 \mathrm{~h}$ under formaldehyde, $96 \mathrm{~h}$ under benzene, $16 \mathrm{~h}$ under TMA, and $48 \mathrm{~h}$ under mixed pollutants). Briefly, IAA was extracted from fresh plant samples ( $4 \mathrm{~g}$ of shoot area) and was purified following the method described in Sinha and Basu (1981). Afterward, the IAA concentration was quantitatively measured following the colorimetric assay of Gordon and Weber (1951). Levels of the phytohormone ethylene was measured at the end of the fumigation period ( $48 \mathrm{~h}$ under formaldehyde, $96 \mathrm{~h}$ under benzene, $16 \mathrm{~h}$ under TMA, and $48 \mathrm{~h}$ under mixed pollutants), following the method described by Kim et al. (2015). The stomata patterns were observed under light conditions (day time) at the end of the formaldehyde fumigation experiment (48 h) (Hultine and Marshall 2001).

\section{Levels of ACC synthase and ACC oxidase activity.}

Samples of plant shoot tissue $(4 \mathrm{~g})$ were obtained at the end of the formaldehyde fumigation experiment (48 h), were thoroughly rinsed with distilled water, and were submerged in ACC synthase reaction buffer (50 mM HEPES, pH 8.5, $0.5 \mu \mathrm{M}$ pyridoxal-5' -phosphate, and $50 \mu \mathrm{M} S$-adenosyI-L-methione) in a 42-ml VOA vial and incubated for $2 \mathrm{~h}$ with $150 \mathrm{rpm}$ shaking. Afterward, a 1-ml gas sample was taken from the headspace of the vial and was analyzed for ethylene levels by gas chromatography. For the ACC oxidase assay, samples were immersed in ACC oxidase reaction buffer (50 mM HEPES, $\mathrm{pH} 8.5,0.5 \mu \mathrm{M}$ pyridoxal-5'-phosphate, and $5 \mathrm{mM} \mathrm{ACC}$ ). Thereafter, ACC oxidase activity was assayed following the protocol of the ACC synthase assay (Song et al. 2007), with brief modifications.

\section{Levels of intracellular reactive oxygen species (ROS).}

Samples of plant shoot tissue $(4 \mathrm{~g})$ were obtained at the end of the fumigation period ( $48 \mathrm{~h}$ under formaldehyde, $96 \mathrm{~h}$ under benzene, $16 \mathrm{~h}$ under TMA, and $48 \mathrm{~h}$ under mixed pollutants) and were ground with pre-chilled mortar and pestle, using liquid nitrogen. Afterward, ground tissue was incubated in medium containing $5 \mathrm{mM} \mathrm{KCl}, 50 \mu \mathrm{M} \mathrm{CaCl}_{2}$, and $10 \mathrm{mM}$ MES-Tris (pH 6.15) for $1 \mathrm{~h}$. Then, homogenate was centrifuged at $4^{\circ} \mathrm{C}$. A $150-\mu \mathrm{l}$ aliquot of supernatant was mixed with $50 \mu \mathrm{l}$ of $50 \mu \mathrm{M} \mathrm{H} \mathrm{H}_{2}$ DCF-DA $\left(2^{\prime}, 7^{\prime}\right.$-dichlorodihydrofluorescein diacetate) and was incubated for $30 \mathrm{~min}$. After incubation, samples were loaded into Corning 96-well plates. Fluorescence intensity was measured using a Tecan infinite M200 microplate reader (Tecan Austria GmbH, Männedorf, Switzerland) with fluorescence excitation and emission at 480 and $520 \mathrm{~nm}$, respectively.

\section{Genomic DNA extraction and identification of endophytic isolates.}

Endophytic isolates were identified by $16 \mathrm{~S}$ rRNA gene sequencing (Khaksar et al. 2016a). The 16S rDNA sequences of the endophytic isolates were deposited in the National Center for Biotechnology Information (NCBI) GenBank database.

\section{Effect of different airborne pollutants on native $\boldsymbol{E}$. milii} inoculated with root endophyte 1 and root endophyte 4 .

Root endophyte 1 and root endophyte 4 were selected for further studies. Native E. milii was inoculated with root endophyte 1 and root endophyte 4 individually through soil to assess the effect of various airborne pollutants with different phytotoxicity levels on plant-endophyte interactions. The fumigation experiment was set as previously described. A 1- $\mu$ l aliquot of liquid benzene (anhydrous, 99.8\%, Sigma-Aldrich) was injected into each chamber to create an initial concentration of $20 \mathrm{ppm}$ gaseous benzene (equal to the initial concentration of gaseous formaldehyde). Gaseous benzene removal (every $12 \mathrm{~h}$ ) and hormonal levels (at the end of fumigation period [96 h]) of noninoculated and endophyte-inoculated plants were measured.

Table 4. Quantitative polymerase chain reaction primers used in this study

\begin{tabular}{|c|c|c|c|}
\hline Isolate & Identification by $16 \mathrm{~S}$ rRNA gene sequencing & Primer $\left(5^{\prime} \rightarrow 3^{\prime}\right)$ & Target gene \\
\hline Root endophyte 1 & Acinetobacter venetianus МТCC11369 & F: GGAGAAAGCAGGGGACCTTC & 16S rRNA \\
\hline Root endophyte 2 & Enterobacter cloacae GGT036 & $\begin{array}{l}\text { R: CAGACCCGCTACAGATCGTC } \\
\text { F: CCGCGCCAATGAAGTTATGG } \\
\text { R: GAATTCCACCATCGCGTCG }\end{array}$ & 16S rRNA \\
\hline Root endophyte 3 & Lysinibacillus fusiformis S5 & $\begin{array}{l}\text { F: AATGGGCGAAAGCCTGATGG } \\
\text { R: GGGCTTTCACATCAGACTTAAAGG }\end{array}$ & 16S rRNA \\
\hline Root endophyte 4 & Citrobacter amalonaticus Y19 & $\begin{array}{l}\text { F: GCTGCTGCGCTTTACTCATC } \\
\text { R: CGGTTCGCAACCATCGATTC }\end{array}$ & 16S rRNA \\
\hline Root endophyte 5 & Pseudomonas putida & $\begin{array}{l}\text { F: ATTAAGTTGACCGCCTGGGG } \\
\text { R: CAGACTGCGATCCGGACTAC }\end{array}$ & 16S rRNA \\
\hline Leaf endophyte 1 & Geobacillus stearothermophilus KS141 & $\begin{array}{l}\text { F: TTGGTGAGGTAACGGCTCAC } \\
\text { R: TACCGTCAAGGTACAGCCAG }\end{array}$ & 16S rRNA \\
\hline Leaf endophyte 2 & Bacillus fusiformis isolate LLP & $\begin{array}{l}\text { F: CAGAGTTTGATCATGGCTCAGG } \\
\text { R: GTGTCTCAGTCCCAGTGTGG }\end{array}$ & 16S rRNA \\
\hline
\end{tabular}


In order to study the effect of TMA, a fumigation experiment was carried out and a 10.7- $\mu$ l aliquot of liquid TMA (40\% solution, Merck, Darmstadt, Germany) was injected into each chamber to create an initial concentration of $100 \mathrm{ppm}$ gaseous TMA. In this part, $100 \mathrm{ppm}$ of gaseous TMA was used in order to be able to assess the difference of TMA removal between noninoculated and endophyte-inoculated E. milii clearly, since E. milii is highly efficient for TMA removal. Furthermore, the effect of mixed-pollutant stress was also investigated by injecting benzene $(1 \mu \mathrm{l})$, formaldehyde $(1.2 \mu \mathrm{l})$, and TMA $(10.7 \mu \mathrm{l})$ into the chamber.

\section{Primer design.}

PCR amplification of 16S rRNA genes from endophytic isolates was performed using the universal primers of $27 \mathrm{f}$ and 1492r. PCR amplicons were purified using a PCR purification kit (QIAquick PCR purification kit; QIAGEN Inc., Valencia, CA, U.S.A.) and were sequenced by Applied Biosystems highest capacity-based genetic analyzer platforms at 1st BASE, Singapore. Identity regions (signature sequences) within the $16 \mathrm{~S}$ rRNA gene for each isolate were selected by multiple alignments of the 16S rRNA gene sequence, using PRIMROSE software (Ashelford et al. 2002). Primers were manually designed based on these selected regions. For primer designing, important parameters, such as GC content, melting temperature, amplicon size, self-complementarity, and potential hairpin formation were examined. Specificity of each designed primer was evaluated using Primer-BLAST (NCBI). The primer sets used in this study are shown in Table 4 . The characteristics of each primer are also summarized in Supplementary Table S3.

\section{Strain-specific qPCR.}

Plant samples (4 $\mathrm{g}$ of shoot area) from each endophyteinoculated and noninoculated E. milii plant in the presence and absence of pollutant were obtained (4 days after inoculation) and were surface-sterilized. Afterward, each surface-sterilized plant sample was macerated and crushed. DNA was extracted from crushed sample using FastDNA Spin Kit for Soil (MP Biomedicals, Santa Ana, CA, U.S.A.), following the manufacturer's instruction. The DNA integrity was then verified using a Nanodrop spectrophotometer (ThermoFisher Scientific, Waltham, MA, U.S.A.) and was stored at $-20^{\circ} \mathrm{C}$ until further use as qPCR template. qPCR was performed using the Agilent Mx3005P QPCR cycler (Santa Clara, CA, U.S.A.). Each qPCR reaction was performed in a total volume of $20 \mu$ l containing $10 \mu \mathrm{l}$ of $2 \times$ KAPA SYBR FAST qPCR master mix universal (Kapa Biosystems, Wilmington, MA, U.S.A.), $0.4 \mu \mathrm{l}$ of each primer $(200 \mathrm{nM}), 0.4 \mu \mathrm{l}$ of $50 \times$ ROX Low (Kapa Biosystems) and $1 \mu \mathrm{l}$ of template DNA ( $20 \mathrm{ng}$ ) in triplicate. qPCR conditions were as follows: an initial denaturation at $95^{\circ} \mathrm{C}$ for $10 \mathrm{~min}, 40$ cycles of denaturation at $95^{\circ} \mathrm{C}$ for $30 \mathrm{~s}$, annealing and extension at $60^{\circ} \mathrm{C}$ for $10 \mathrm{~s}$, and $72^{\circ} \mathrm{C}$ for $20 \mathrm{~s}$, followed by one cycle of $95^{\circ} \mathrm{C}$ for $1 \mathrm{~min}, 55^{\circ} \mathrm{C}$ for $30 \mathrm{~s}$, and $95^{\circ} \mathrm{C}$ for $30 \mathrm{~s}$. Amplification efficiency for each qPCR assay was calculated as follows:

$$
E=\left(10^{\frac{1}{- \text { slope }}}-1\right) * 100,
$$

where $E$ is amplification efficiency and slope was calculated from plotting log transformation of serially diluted target gene copy number $\left(10^{8}\right.$ to $\left.10^{2}\right)$ versus cycle threshold. qPCR descriptors have been reported in Supplementary Table S4.

\section{Gas chromatography analysis.}

In order to analyze remaining concentrations of gaseous pollutants, gas chromatography analysis was done using a gas chromatography with flame ionization detector (GC-FID), model GC-430 from Bruker (Billerica, MA, U.S.A.). The experimental conditions for GC-FID included a $100^{\circ} \mathrm{C}$ injection temperature, $130^{\circ} \mathrm{C}$ column temperature, and $150^{\circ} \mathrm{C}$ detector temperature.

\section{Statistical analysis.}

The statistical analysis of the data were conducted by a oneway analysis of variance (ANOVA) and Duncan's multiple range tests, using the SPSS software version 20 with a $95 \%$ confidence level. ANOVA was used to calculate mean difference of values with Duncan's multiple range tests to classify the group of data.

\section{ACKNOWLEDGMENTS}

The authors thank the Higher Education Research Promotion and $\mathrm{Na}-$ tional Research University Project of Thailand, Office of the Higher Education Commission and King Mongkut's University of Technology Thonburi (Petchra Pra Jom Klao Ph.D. scholarship, grant number 10/2557) for financially supporting G. Khaksar.

\section{LITERATURE CITED}

Abeles, F. B., Morgan, P. W., and Saltveit, M. E., Jr. 1992. Ethylene in plant biology, 2nd ed. Academic Press, New York.

Adams, D. O., and Yang, S. F. 1979. Ethylene biosynthesis: Identification of 1-aminocyclopropane-1-carboxylic acid as an intermediate in the conversion of methionine to ethylene. Proc. Natl. Acad. Sci. U.S.A. 76: $170-174$.

Afzal, M., Khan, Q. M., and Sessitsch, A. 2014. Endophytic bacteria: Prospects and applications for the phytoremediation of organic pollutants. Chemosphere 117:232-242.

Ashelford, K. E., Weightman, A. J., and Fry, J. C. 2002. PRIMROSE: A computer program for generating and estimating the phylogenetic range of 16S rRNA oligonucleotide probes and primers in conjunction with the RDP-II database. Nucleic Acids Res. 30:3481-3489.

Babu, A. G., Kim, J. D., and Oh, B. T. 2013. Enhancement of heavy metal phytoremediation by Alnus firma with endophytic Bacillus thuringiensis GDB-1. J. Hazard. Mater. 250-251:477-483.

Barac, T., Taghavi, S., Borremans, B., Provoost, A., Oeyen, L., Colpaert, J. V., Vangronsveld, J., and van der Lelie, D. 2004. Engineered endophytic bacteria improve phytoremediation of water-soluble, volatile, organic pollutants. Nat. Biotechnol. 22:583-588.

Bell, C. R., Dickie, G. A., Harvey, W. L. G., and Chan, J. W. Y. F. 1995. Endophytic bacteria in grapevine. Can. J. Microbiol. 41:46-53.

Chen, Y. F., Etheridge, N., and Schaller, G. E. 2005. Ethylene signal transduction. Ann. Bot. (Lond.) 95:901-915.

Chen, Z. J., Sheng, X. F., He, L. Y., Huang, Z., and Zhang, W. H. 2013. Effects of root inoculation with bacteria on the growth, Cd uptake and bacterial communities associated with rape grown in Cd-contaminated soil. J. Hazard. Mater. 244-245:709-717.

Chhon, Y. 2013. Phytoremediation of Trimethylamine by Euphorbia milii and agricultural residues. Master's thesis, King Mongkut's University of Technology Thonburi, Bangkok.

Daszkowska-Golec, A., and Szarejko, I. 2013. Open or close the gate stomata action under the control of phytohormones in drought stress conditions. Front. Plant Sci. 4:138.

Fei, Y., Xiao, B., Yang, M., Ding, Q., and Tang, W. 2016. MicroRNAs, polyamines, and the activities antioxidant enzymes are associated with in vitro rooting in white pine (Pinus strobus L.). Springerplus 5:416.

Glick, B. R. 2014. Bacteria with ACC deaminase can promote plant growth and help to feed the world. Microbiol. Res. 169:30-39.

Gordon, S. A., and Weber, R. P. 1951. Colorimetric estimation of indoleacetic acid. Plant Physiol. 26:192-195.

Guan, L. M., and Scandalios, J. G. 2002. Catalase gene expression in response to auxin-mediated developmental signals. Physiol. Plant. 114: 288-295.

Ho, Y. N., Mathew, D. C., Hsiao, S. C., Shih, C. H., Chien, M. F., Chiang, H. M., and Huang, C. C. 2012. Selection and application of endophytic bacterium Achromobacter xylosoxidans strain F3B for improving phytoremediation of phenolic pollutants. J. Hazard. Mater. 219-220:43-49.

Hultine, K. R., and Marshall, J. D. 2001. A comparison of three methods for determining the stomatal density of pine needles. J. Exp. Bot. 52: 369-373. 
Khaksar, G., Treesubsuntorn, C., and Thiravetyan, P. 2016a. Endophytic Bacillus cereus ERBP-Clitoria ternatea interactions: Potentials for the enhancement of gaseous formaldehyde removal. Environ. Exp. Bot. 126:10-20.

Khaksar, G., Treesubsuntorn, C., and Thiravetyan, P. 2016b. Effect of endophytic Bacillus cereus ERBP inoculation into non-native host: Potentials and challenges for airborne formaldehyde removal. Plant Physiol. Biochem. 107:326-336.

Kim, Y. H., Hwang, S. J., Waqas, M., Khan, A. L., Lee, J. H., Lee, J. D., Nguyen, H. T., and Lee, I. J. 2015. Comparative analysis of endogenous hormones level in two soybean (Glycine $\max$ L.) lines differing in waterlogging tolerance. Front. Plant Sci. 6:714.

Kobayashi, D. Y., and Palumbo, J. D. 2000. Bacterial endophytes and their effects on plants and uses in agriculture. Pages 199-233 in: Microbial endophytes. Bacon, C. W., and White, J. F., eds. Marcel Dekker, Inc., New York.

Li, J., McConkey, B. J., Cheng, Z., Guo, S., and Glick, B. R. 2013. Identification of plant growth-promoting bacteria-responsive proteins in cucumber roots under hypoxic stress using a proteomic approach. J. Proteomics 84:119-131.

Mayak, S., Tirosh, T., and Glick, B. R. 2004. Plant growth-promoting bacteria confer resistance in tomato plants to salt stress. Plant Physiol. Biochem. 42:565-572.

Patriquin, D. G., and Döbereiner, J. 1978. Light microscopy observations of tetrazolium-reducing bacteria in the endorhizosphere of maize and other grasses in Brazil. Can. J. Microbiol. 24:734-742.

Rajkumar, M., Ae, N., and Freitas, H. 2009. Endophytic bacteria and their potential to enhance heavy metal phytoextraction. Chemosphere 77:153-160.

Sinha, B. K., and Basu, P. S. 1981. Indole-3-acetic acid metabolism in root nodules of Pongamia pinnata (L.). Biochem. Physiol. Pflanz. 176:218-227.
Siswanto, D., Chhon, Y., and Thiravetyan, P. 2016. Uptake and degradation of trimethylamine by Euphorbia milii. Environ Sci Pollut Res. Int. Published online. doi:10.1007/s11356-016-6874-Z

Song, Y. J., Joo, J. H., Ryu, H. Y., Lee, J. S., Bae, Y. S., and Nam, K. H. 2007. Reactive oxygen species mediate IAA-induced ethylene production in mungbean (Vigna radiata L.) hypocotyls. J. Plant Biol. 50: 18-23.

Sriprapat, W., Boraphech, P., and Thiravetyan, P. 2014. Factors affecting xylene-contaminated air removal by the ornamental plant Zamioculcas zamiifolia. Environ. Sci. Pollut. Res. Int. 21:2603-2610.

Sriprapat, W., and Thiravetyan, P. 2013. Phytoremediation of BTEX from indoor air by Zamioculcas zamiifolia. Water Air Soil Pollut. 224: 1482.

Torres, M. A., Dangl, J. L., and Jones, J. D. G. 2002. Arabidopsis gp91phox homologues AtrbohD and AtrbohF are required for accumulation of reactive oxygen intermediates in the plant defense response. Proc. Natl. Acad. Sci. U.S.A. 99:517-522.

Treesubsuntorn, C., and Thiravetyan, P. 2012. Removal of benzene from indoor air by Dracaena sanderiana: Effect of wax and stomata. Atmos. Environ. 57:317-321.

Visioli, G., D’Egidio, S., Vamerali, T., Mattarozzi, M., and Sanangelantoni, A. M. 2014. Culturable endophytic bacteria enhance Ni translocation in the hyperaccumulator Noccaea caerulescens. Chemosphere 117:538544.

Xie, H., Pasternak, J. J., and Glick, B. R. 1996. Isolation and characterization of mutants of the plant growth-promoting rhizobacterium Pseudomonas putida GR12-2 that overproduce indoleacetic acid. Curr. Microbiol. 32:67-71. 